\title{
CERTAIN THEOREMS RELATING TO PLANE CONNECTED POINT SETS*
}

BY

\author{
ANNA M. MULLIKIN
}

\section{INTRODUCTION}

A point set $M$ is said $\dagger$ to be connected if it cannot be expressed as the sum of two mutually exclusive point sets neither of which contains a limit point of the other. Sierpinskif has shown that a closed, bounded, connected set of points in space of $n$ dimensions cannot be separated into a countable infinity of closed point sets such that no two of them have a point in common. It will be shown in the present paper that for the case where $n=2$, this theorem does not remain irue if the stipulation that $M$ is closed be removed. It will however be shown that a plane point set, regardless of whether it be closed or bounded, which separates its plane cannot be expressed as the sum of a countable infinity of closed, mutually exclusive point sets, no one of which separates the plane. Of the other results established, the principal one is that if $M_{1}$ and $M_{2}$ are two closed, connected, bounded point sets, neither of which disconnects a plane $S$, a necessary and sufficient condition that their sum, $M$, shall disconnect $S$ is that $\bar{M}$, the set of points common to $M_{1}$ and $M_{2}$, be not connected.

I wish to thank Professor Robert L. Moore, who suggested the theorems of this paper. Without his help and encouragement it could not have been written.

\section{II}

The following is an example of a countable collection of mutually exclusive, closed, and bounded point sets with connected sum. Consider a countable infinity of arcs each of which is made up of four straight-line intervals (Fig. 1), the $n$th arc being drawn from the point $\left(m / 2^{n-1}, 0\right)$ to $\left(m / 2^{n-1}, m / 2^{n-1}\right)$, thence to $\left(-m / 2^{n-1}, m / 2^{n-1}\right)$, thence to $\left(-m / 2^{n-1},-m / 2^{n-1}\right)$ and thence to $\left(m,-m / 2^{n-1}\right)$. Let $n$ go from one to infinity, and let $M$ be the point

* Various parts of this paper were presented to the Society on October 25, 1919, December 28, 1920, and February 26, 1921.

$\dagger$ See N. J. Lennes, Curves in non-metrical analysis situs with an application in the calculus of variations, American Journal of Mathematics, vol. 33 (1911), and Bulletin of the American Mathematical Society, vol. 12 (1906).

$\ddagger \mathrm{W}$. Sierpinski, Un théorème sur les continus, $\mathrm{T}$ ô h o $\mathrm{k} u \mathrm{M}$ a t h e $\mathrm{m}$ a t i c a l J o u rn al, vol. 13. 
set composed of the sum of all the arcs so obtained. It will be seen that each of these arcs contains a limit point of every subset of $M$ which consists of an infinite number of the remaining arcs. Hence the set $M$ is connected. It is obviously bounded.

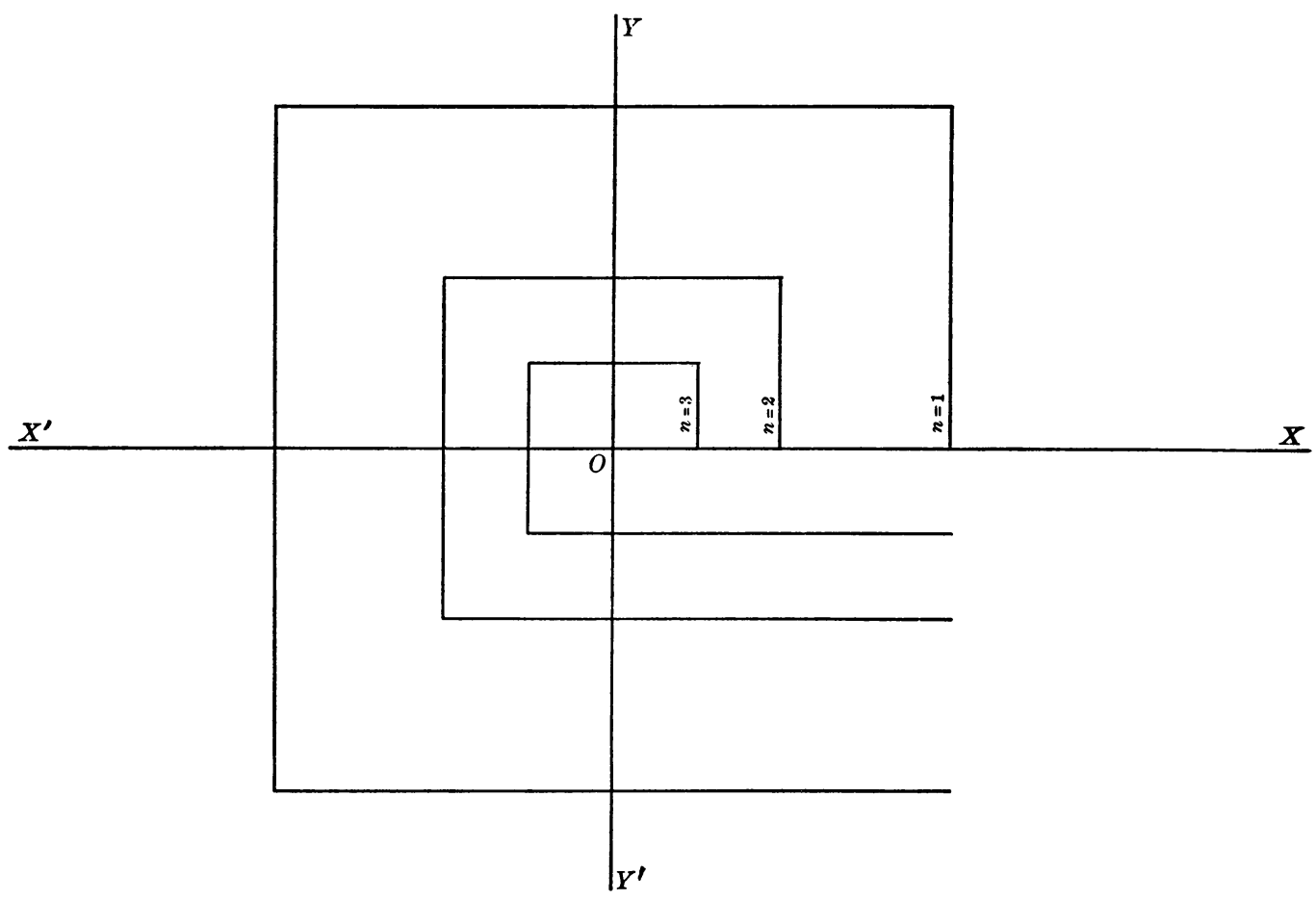

FIg. 1

Theorem 1.* If, in a plane $S, K$ and $M$ are two closed, mutually exclusive point sets and $H$ is a closed, bounded, connected point set having at least one point in common with each of the sets $K$ and $M$, then there exists a point set $\bar{H}$, a subset of $H$, such that $\bar{H}$ is connected and contains no point of either $K$ or $M$, but such that $K$ and $M$ each contain a limit point of $\bar{H}$.

In our proof of Theorem 1, we shall make use of the following two well known theorems, $A$ and $B$.

Theorem $A . \dagger$ If $K$ and $M$ are two closed point sets having no point in common, and $H$ is a continuous, bounded point set having at least one point in

* Rosenthal gives a proof for the case in which each of the sets $K$ and $M$ reduces to a single point. See A. Rosenthal, Teilung der Ebene durch irreduzible Kontinua, S it zu ng sberichte der mathematisch-physikalischen Klasseder Bayerischen Akademie der Wissenschaften zu München, 1919, p. 104.

$\dagger$ Janiszewski gives a proof for the case in which each of the sets $K$ and $M$ reduces to a single point. His proof can readily be extended to the more general case. Cf. S. Janiszewski, Sur les continus irréductibles entre deux points, Journal de L'E cole Polytech$\mathrm{n}$ i q u e (2), vol. 16 (1912), p. 109, Theorem 1. 
common with each of the sets $K$ and $M$, then $H$ contains a subset which is irreducibly continuous from $K$ to $M$.

Theorem $B .^{*}$ If $H_{1}, H_{2}, H_{3}, \cdots$ is a countable collection of connected point sets, and $P$ is a point such that every circle containing $P$ contains a point from all except a finite number of these sets, then the limit set $\dagger$ of the sequence $\mathrm{H}_{1}, \mathrm{H}_{2}, \mathrm{H}_{3}, \cdots$ is connected.

Proof of Theorem 1. By Theorem $A, H$ contains a subset which is irreducibly continuous from $K$ to $M$. Call this set $H_{K M}$. Let $\bar{K}$ and $\bar{M}$ denote the points of $H_{K M}$ belonging to $K$ and $M$ respectively. Let $H^{\prime}$ denote $H_{K M}-\bar{K}-\bar{M}$. We can show that $H^{\prime}$ is the required $\bar{H}$. Evidently it only remains to be proved that $H^{\prime}$ is connected. Consider any point $P$ of $H^{\prime}$. We can show that the largest connected subset of $H^{\prime}$ in which $P$ lies has a limit point in either $\bar{K}$ or $\bar{M}$. For suppose it has not. It will then be closed and may be enclosed in a finite number of circles no one of which contains or encloses a point of either $\bar{K}$ or $\bar{M}$. The interiors of these circles form a domain $D_{P}$. Now select some point $K_{1}$ of $\bar{K}$. Since $P$ and $K_{1}$ lie together in the connected set $H_{K M}$ they can be joined by a broken line composed of a finite number of intervals of length less than half an inch, such that the vertices of this broken line belong to $H_{K M}$. Let $L_{1}$ be that vertex on this broken line which immediately precedes the first vertex on it, in the order from $P$ to $K_{1}$, that lies without $D_{P}$. Then join $P$ and $K_{1}$ by a broken line of intervals of length less than a quarter of an inch such that the vertices belong to $H_{K M}$. Let $L_{2}$ be the point on this line corresponding to $L_{1}$. Continue this process indefinitely. By Theorem $B$ the limit set will be connected. It will contain $P$ and a point on the boundary of $D_{P}, \ddagger$ namely the limit point of $L_{1}, L_{2}, L_{3}, \cdots$. It contains only points of $H_{K M}$, but since it lies wholly within $D_{P}$ plus its boundary, it contains no point of $\bar{K}$ or $\bar{M}$ and hence is a subset of $H^{\prime}$. This is contrary to the hypothesis that the largest connected subset of $H^{\prime}$ in which $P$ lies is within the domain $D_{P}$.

Denote by $H_{K}$ the set of those points lying in a connected subset of $H^{\prime}$ of which $\bar{K}$ contains a limit point, by $H_{M}$ the set of those which lie in a connected subset of $H^{\prime}$ of which $\bar{M}$ contains a limit point. Let $H_{K}+\bar{K}$ be denoted by $S_{K}, H_{M}+\bar{M}$ by $S_{M}$. Since $S_{K}+S_{M}=H_{K M}$, and since $H_{K M}$ is connected, $S_{K}$ and $S_{M}$ must have a point in common or else one of these sets must contain a limit point of the other. Suppose first that they have a point in common. This point must belong to $H^{\prime}$, and it is evident that since $H_{K M}$ is irreducibly

* See S. Janiszewski, loc. cit., p. 98, Theorem 1.

† By the limit set of a sequence of sets $H_{1}, H_{2}, H_{3}, \cdots$ we mean the set of all points $\left[P\right.$ ] such that $P$ is a limit point of a set of points $X_{1}, X_{2}, X_{3}, \cdots$ such that for every $k, X_{k}$ belongs to $H_{k}$.

¥ Janiszewski gives a parallel argument to prove that if the continuous set $C$ contains a point $A$ which is an interior point of the closed set $K$, then there exists a continuous set containing the point $A$ and contained in $K$ and $C$. See S. Janiszewski, loc. cit., p. 100, Theorem IV. 
continuous, $H^{\prime}$ must in this case be connected. Suppose secondly that one of the sets contains a limit point of the other, for instance that $S_{K}$ contains a limit point, $P_{K}$, of $S_{M}$. And suppose that $H^{\prime}$ is not connected. Since $S_{K}$ contains a limit point of $S_{M}$ it is evident that $H_{M}$ must exist. Then if $H_{M}=H^{\prime}$, $H_{M}$ cannot be connected. But suppose that the set $H_{K}$ actually exists; we can show that in this case, too, $H_{M}$ is not connected. For suppose it were. $P_{K}+H_{M}$ together with the largest connected subset of $H^{\prime}$ in which $P_{K}$ lies and all points in $H_{K}$ which are limit points of $H_{M}$ would then be a connected subset of $H^{\prime}$ and consequently a proper subset of $H^{\prime}$. This together with its limit points in $\bar{K}$ and $\bar{M}$ would be both a continuous set between $K$ and $M$ and a proper subset of $H_{K M}$. This is contrary to the hypothesis that $H_{K M}$ is irreducibly continuous between $K$ and $M$. We have therefore shown that if $H^{\prime}$ is not connected, $H_{M}$ is not connected.

Suppose this to be the case, and let $H_{M}=H_{M_{1}}+H_{M_{2}}$ where $H_{M_{1}}$ and $H_{M_{2}}$ are two mutually exclusive sets neither of which contains a limit point of the other. Suppose $P_{K}$ is a limit point of $H_{M_{1}}$. Then enclose every point of $H_{M_{2}}$ in a circle which encloses no point of the set $H_{M_{1}}+P_{K}+\bar{M}$. The interiors of these circles form a domain $D$. Now since $P_{K}$ is a limit point of $H_{M_{1}}$ and every point of $H_{M_{1}}$ is connected with some point of $\bar{M}$ in a subset of $H_{M_{1}}+\bar{M}$, $P_{K}$ can be joined by an infinite number of broken lines, as before, to points of $\bar{M}$ such that the vertices of these broken lines belong to $H_{M_{1}}+\bar{M}$, and therefore lie without $D$ or on its boundary. The limit set will then be connected and will contain no point of $D$. This together with the largest connected subset of $H^{\prime}$ in which $P_{K}$ lies and the limit points of this set in $\bar{K}$ will be a continuous set from $K$ to $M$, a proper subset of $H_{K M}$ since it contains no point of $H_{M_{2}}$. This is contrary to the hypothesis that $H_{K M}$ is irreducibly continuous from $K$ to $M$. We have therefore proved that $H^{\prime}$ is connected and is the required $\bar{H}$.

Theorem 2. If, in a plane $S, H$ is a closed, bounded point set containing two mutually exclusive, closed point sets $K$ and $M$, but containing no closed, connected subset containing a point of $K$ and a point of $M$, then it is the sum of two mutually exclusive, closed sets, of which one contains $K$ and the other contains $M$.

Proof. There exists a positive number $\epsilon$ such that no point of $K$ can be joined to a point of $M$ by a broken line made up of intervals of length less than $\epsilon$ such that the end points of these intervals are points of $H$. For otherwise there would be a closed, connected " limit set" as in Theorem 1. This limit set would belong to $H$, since $H$ is closed, and it would contain a point of $K$ and a point of $M$, since $K$ and $M$ are both closed. This is contrary to the hypothesis.

Now let $H_{1}$ denote the point set composed of $K$ together with the set of all 
points [ $P$ ] of $H$ such that $P$ can be connected with some point of $K$ by a broken line of intervals of length less than $\epsilon$ such that the end points of these intervals belong to $H$. Let $H_{2}$ denote the point set composed of all other points of $H . \quad H_{2}$ will contain $M$ and it can easily be seen that neither $H_{1}$ nor $\mathrm{H}_{2}$ contains a limit point of the other, since every point of $\mathrm{H}_{2}$ is at a distance greater than or equal to $\epsilon$ from every point of $H_{1}$.

Lemma. If $M$ is a closed set not disconnecting* a plane $S$ then any two points of $S-M$ can be joined by a simple continuous arc lying in $S-M$.

Proof. Let $P$ denote any point of $S-M$. Let $S_{1}$ denote the point set composed of $P$ together with all points that can be joined to $P$ by a simple continuous arc lying in $S-M$. Let $S_{2}$ denote the set $S-M-S_{1}$, and suppose that $S_{2}$ contains at least one point. Now since $M$ does not disconnect $S, S_{1}$ contains a limit point of $S_{2}$, or $S_{2}$ of $S_{1}$. Suppose that $S_{1}$ contains a limit point $P_{1}$ of $S_{2}$. Then enclose $P_{1}$ within a square $K$ which neither contains not encloses a point of $M$. This square will enclose a point $P_{2}$ of $S_{2}$. Then $P_{1}$ and $P_{2}$ can be joined by a straight line interval lying within $K$ and therefore containing no point of $M$. Since $P_{1}$ can be joined to $P$ by a simple continuous arc lying in $S-M$ it is obvious that $P_{2}$ can also. The argument would be similar in the case where $S_{2}$ contains a limit point of $S_{1}$. Since either leads to a contradiction we have proved that $S_{2}$ does not contain even one point.

THEOREM 3. If $M$ is the sum of a countable number of closed, mutually exclusive point sets $M_{1}, M_{2}, M_{3}, \cdots$, no one of which disconnects a plane $S$, then $M$ does not disconnect $S$.

Proof. Suppose on the contrary that $S-M=S_{1}+S_{2}$, where $S_{1}$ and $S_{2}$ are mutually exclusive and neither contains a limit point of the other. Let $\bar{M}_{1}$ denote a point set composed of those points of $M$ that are limit points of $S_{1}$ but not of $S_{2}, \bar{M}_{2}$ the point set composed of those that are limit points of $S_{2}$, but not of $S_{1}$, and let $\bar{M}$ denote the point set composed of those points of $M$ that are limit points of neither $S_{1}$ nor $S_{2}$. There exists in $S$ a countable collection $K_{P_{1}}$ of squares $K_{1}, K_{2}, K_{3}, \cdots$ obtained in the following manner. Take any point $P_{1}$ of $S_{1}$ as the center of a square $\bar{K}_{1}$ of side 2 inches. Let $K_{1}, K_{2}, K_{3}, K_{4}$ be the four squares of side one inch each contained in $\bar{K}_{1}$ and taken in any order. In general let $\bar{K}_{n}$ be a square of side $2^{n}$ inches which has $P_{1}$ for its center and has its sides parallel to those of $\bar{K}_{1}$, and let it be divided into $2^{4 n-2}$ squares, each of side $1 / 2^{n-1}$ inches, and let these $2^{4 n-2}$ squares follow each other in any order, and let them be the squares $\frac{K^{2+m-2}+11}{15}, \cdots, \frac{K^{2+2+2-4}}{15}$ in the set $K_{P_{1}}$.

Now consider the first square $K_{1}^{\prime}$ of $K_{P_{1}}$ which contains $P_{1}$ and satisfies

${ }^{*} M$ is said to disconnect $S$ if $S-M$ is the sum of two mutually exclusive point sets neither of which contains a limit point of the other. 
condition (1) that it contain and enclose only points of $S_{1}+\bar{M}_{1}+\bar{M}$. There evidently exists one such square, since $P_{1}$ is not a limit point of $S_{2}$. Add to $K_{1}^{\prime}$ the first square $K_{2}^{\prime}$ of $K_{P_{1}}$ that encloses no point of the interior of $K_{1}^{\prime}$ and that satisfies condition (1) and also condition (2) that it shall contain or enclose at least one point of $S_{1}$, and condition (3) that it shall have an interval in common with $K_{1}^{\prime}$. It is evident that there exists a simple closed curve $C_{2}$ which is a subset of $K_{1}^{\prime}+K_{2}^{\prime}$ and such that the interiors of $K_{1}^{\prime}$ and $K_{2}^{\prime}$ are subsets of the interior of $C_{2}$. In general obtain $C_{3}, C_{4}, C_{5}, \ldots$ in the following manner: $C_{r}$ shall be obtained by adding to $C_{r-1}$ the first square $K_{r}^{\prime}$ of $K_{P_{1}}$ which encloses no point of the interior of $C_{r-1}$ and which satisfies conditions (1) and (2) and contains an interval in common with $C_{r-1}$. Then $C_{r}$ is a simple closed curve which is a subset of $C_{r-1}+K_{r}^{\prime}$ and whose interior contains the interiors of $C_{r-1}$ and $K_{r}^{\prime}$. It can easily be shown that provided $K_{r}^{\prime}$ exists and $C_{r-1}$ can be obtained in this manner, then $C_{r}$ can also. For let $A_{1} A_{2}$ be an interval common to $C_{r-1}$ and $K_{r}^{\prime}$. Let $A_{1} \bar{B} A_{2}$ (Fig. 2) be an arc

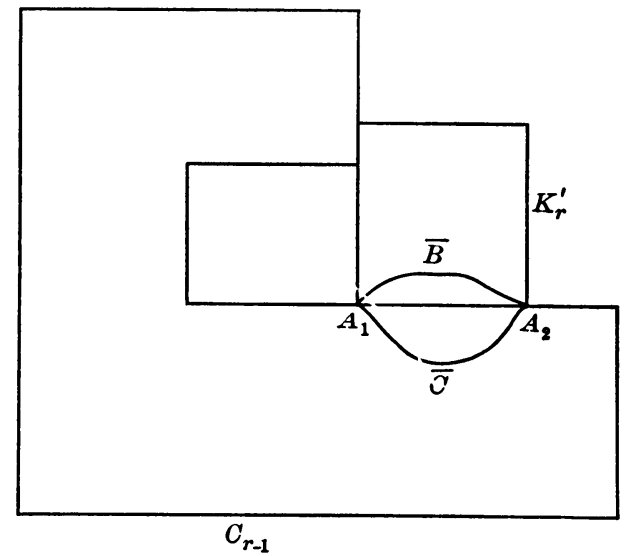

FIG. 2.

lying except for its end points within $K_{r}^{\prime}$ and let $A_{1} \bar{C} A_{2}$ be an arc lying except for its end points within $C_{r-1}$. Then in Theorem 41 of The foundations of plane analysis situs, ${ }^{*}$ let $J_{1}$ be $C_{r-1}-A_{1} A_{2}+A_{1} \bar{B} A_{2}$ and let $J_{2}$ be $K_{r}^{\prime}-A_{1} A_{2}+A_{1} \bar{C} A_{2}$. Then $J$ will be the required $C_{r}$. Denote by $C$ the set of curves $C_{1}\left(K_{1}^{\prime \prime}\right) C_{2}, C_{3}, \cdots$.

The sequence $C$ will evidently be infinite unless some $C_{i}$ contains only points of $M$. Suppose this to be the case, and suppose first that there is some point $P_{E}$ of $S_{1}$ or $S_{2}$ without $C_{i}$. The curve $C_{i}$ divides $S$ into two parts, its interior $I$, and its exterior $E$, such that neither of these parts contains a limit point of the other. Now all of $C_{i}$ must belong to the same set in $M$,

${ }^{*}$ See R. L. Moore, On the foundations of plane analysis situs, these Trans a ct.i o n s, vol. 17 (1916), p. 155. 
say $M_{j}$, since there does not exist a countable collection of closed, mutually exclusive point sets, consisting of more than one set, whose sum is closed, bounded, and connected. $\dagger$ Then $M_{j}$ disconnects $S$; one part of $S-M_{j}$ is composed of those points of $I$ which do not belong to $M_{j}$ ( $P_{1}$ is one such point); the other part is composed of those points of $E$ which do not belong to $M_{j}$ (by hypothesis there is at least one such point). But this is contrary to the hypothesis of the theorem. Suppose, secondly, that all points of $C_{i}$ and its exterior belong to $M_{j}$. Start from $P_{1}$ again to add squares of $K_{P_{1}}$, this time adding the extra condition that no square added shall contain or enclose a point of $C$ or its exterior or of $M_{j}$; this is possible since $M_{j}$ and the set composed of $C_{i}$ together with its exterior are closed sets. Suppose again that some $C_{k}$ of this new set of curves is a subset of $M$, and therefore of, say, $M_{l}$. If there is some point of $S_{1}$ or $S_{2}$ without $C_{k}$, then, as above, $M_{l}$ disconnects $S$, which is contrary to hypothesis. But there must be a point of $S_{1}$ or $S_{2}$ in the closed, connected, bounded point set made up of $C_{i}+C_{k}$ and all points between them, as otherwise this set of points would be the sum of a countable collection of closed, mutually exclusive point sets, subsets of $M$, and would contain subsets of at least two of the sets $M_{1}, M_{2}, M_{3}, \cdots$, namely of $M_{j}$ and of $M_{l}$. Thus in any case we obtain an infinite sequence $C$, every curve of which contains at least one point of $S_{1}$ and contains only points of $S_{1}+\bar{M}_{1}+\bar{M}$. Let $D_{1}$ denote the sum of the interiors of these curves.

Now suppose there is no point of $S_{2}$ without $D_{1}$. Then take a point $P_{2}$ of $S_{2}$ that is within some curve $C_{P_{2}}$ of $C$ and add squares to it in the manner in which we obtained $K_{1}^{\prime}, K_{2}^{\prime}, K_{3}^{\prime}, \cdots$, except that these squares with their interiors are subsets of $S_{2}+\bar{M}_{2}+\bar{M}$ and each one contains or encloses at least one point of $S_{2}$, and add the extra condition that no one shall contain a point of $C_{P_{2}}$ or of its exterior. The sum of the interiors of the curves so obtained will be a domain $D_{2}$, a subset of the interior of $C_{P_{2}}$ and therefore not containing all points of $S_{1}$. Since $D_{2}$ would serve as well for the argument as $D_{1}$ we shall suppose that not every point of $S_{2}$ is within $D_{1}$.

Then since $D_{1}$ does not contain all of $S$ it must have some boundary points; let $B$ denote the boundary of $D_{1}$. Suppose $B$ contains a point $P_{1}^{\prime}$ of $S_{1}$. There is a square $\bar{R}$ of the sequence $K_{P_{1}}$ which encloses or contains $P_{1}^{\prime}$ but no point or limit point of $S_{2}$ and which encloses a point of a curve of the sequence $C$. Consider the first curve of $C$ which was obtained by adding a square having an interval in common with $\bar{R}$ or its interior. Let $R^{*}$ be the square so added. Consider two cases.

Case I. It is given that $R^{*}$ has an interval in common with $\bar{R}$. If the interiors of these squares are mutually exclusive, $\bar{R}$ possesses all of the properties necessary for it to be added in obtaining some curve of $C$, and it will

† Cf. M. Sierpinski, loc. cit. 
subsequently be added or enclosed by the addition of some other square of $K_{P_{1}}$. If the interiors of $R^{*}$ and $\bar{R}$ are not mutually exclusive, evidently the interior of $\bar{R}$ must include that of $R^{*}$. But $\bar{R}$ precedes $R^{*}$ in the sequence $K_{P_{1}}$ and would have been used instead of $R^{*}$, since $\bar{R}$ contains an interval of $R^{*}$ that $R^{*}$ has in common with that curve of $C$ to which we supposed it added.

Case II. It is given that $R^{*}$ has an interval in common with the interior of $\bar{R}$. In this case the interior of $R^{*}$ lies wholly within $\bar{R}$ but obviously they must also have an interval in common, since $R^{*}$ was the square added to obtain the first curve of $C$ having an interval in common with $\bar{R}$ or its interior. The argument is the same then as in Case I. We have therefore proved that $B$ contains no point of $S_{1}$.

Furthermore $B$ contains no point of $S_{2}$. For suppose $P_{2}^{\prime}$ is a point of $S_{2}$ belonging to $B$. It is not on any curve of $C$; therefore it must be a limit point of an infinite number of curves of $C$. Since $P_{2}^{\prime}$ is not a limit point of $S_{1}$ there exists a square $K_{h}$ with $P_{2}^{\prime}$ as center, which neither contains nor encloses a point of $S_{1}$. Suppose a side of $K_{h}$ is $\epsilon_{1}$ inches long. There exists only a finite number of squares of $K_{P_{1}}$ of side equal to or greater than $\epsilon_{1} / 8$ that have points in common with $K_{h}$ or its interior. Let $Q$ denote this set of squares. If any square of $Q$ was used in the sequence $K_{1}^{\prime}, K_{2}^{\prime}, K_{3}^{\prime}, \cdots$, let $K_{n}^{\prime}$ denote the last square in this sequence that belongs to $Q$. Then $C_{n}$ will be the last curve of $C$ formed by adding a square of $Q$. If no square of $Q$ was so used, let $C_{n}$ denote any curve of $C$. Then $K_{h}$ encloses a point $P_{l}$ which lies on no curve of the set $C_{1}, C_{2}, \cdots, C_{n}$, but which does lie on a curve of $C$ following $C_{n}$ and such that the distance from $P_{2}^{\prime}$ to $P_{l}$ is less than $\epsilon_{1} / 4$. Then $P_{l}$ must be a point on a square of the sequence $K_{1}^{\prime}, K_{2}^{\prime}, K_{3}^{\prime}, \cdots$ of side less than $\epsilon_{1} / 8$ and therefore is at a distance of less than $\epsilon_{1} / 4$ from a point of $S_{1}$, since every square of the sequence $K_{1}^{\prime}, K_{2}^{\prime}, K_{3}^{\prime}, \cdots$ contains or encloses a point of $S_{1}$. This point of $S_{1}$ would then lie within $K_{h}$, which leads to a contradiction. We have therefore proved that $B$ is a subset of $M$.

We can now prove that two closed, mutually exclusive point sets neither of which disconnects $S$ cannot together disconnect $S . \dagger$ For suppose $M=M_{1}+M_{2}$. We have shown above that not every point of $S_{2}$ is in $D_{1}$ and that no point of $S_{2}$ is on $B$. Let $P_{0}$ denote a point of $S_{2}$ without $D_{1}$. There is a simple continuous arc from $P_{0}$ to $P_{1}$. Let $B_{1}$ denote the first point of $B$ on this arc in the order $P_{0} P_{1}$. Suppose that $M_{1}$ is that one of the sets $M_{1}$ and $M_{2}$ to which $B_{1}$ belongs. Since $M_{1}$ is a closed set not disconnecting $S, P_{0}$ can, by the lemma, be joined to $P_{i}$ by an arc not containing any point of $M_{1}$. Let $B_{2}$ be the first point of $B$ on this arc $\neq$ in the order $P_{0} P_{1}$. Then

$\dagger$ Hausdorff gives a proof for the case when one of the sets is bounded. Cf. F. Hausdorff, Grundzüge der Mengenlehre, Leipzig, Veit, 1914, p. 342.

$\ddagger$ Hereafter in this paper, "arc" and "simple continuous arc" will be considered synonymous terms. 
$B_{2}$ belongs to $M_{2}$. The set $P_{0} B_{1}+P_{0} B_{2}$ contains as a subset an $\operatorname{arc} B_{1} B_{2}$. Let $H_{1}$ be a simple closed curve enclosing $B_{1}$ but neither containing nor enclosing any point of $M_{2}$ (Fig. 3) and containing only one point $L_{1}$ of $B_{1} B_{2}$,

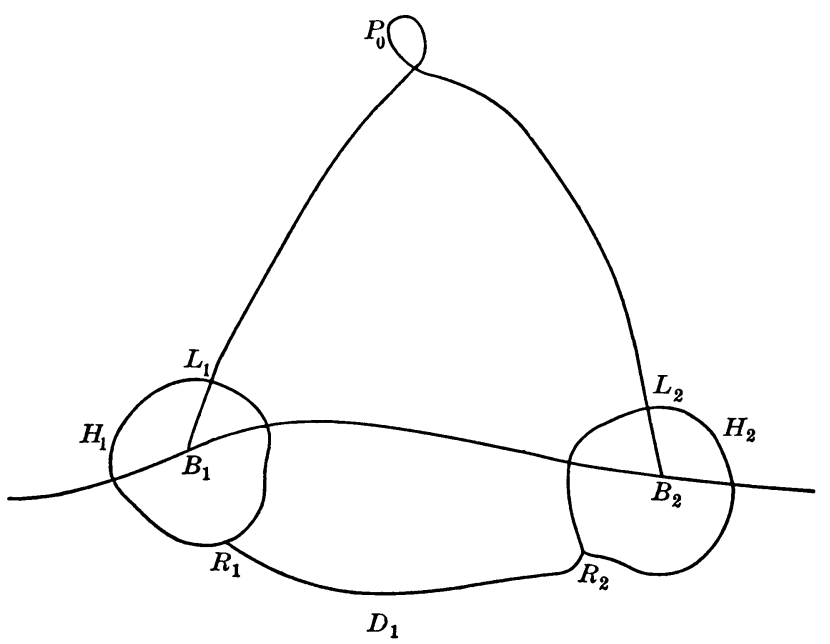

FIG. 3.

and let $H_{2}$ be a simple closed curve neither containing nor enclosing any point of $H_{1}$ or $M_{1}$, but enclosing $B_{2}$ and containing only one point $L_{2}$ of $B_{1} B_{2}$. Let a point of $D_{1}$ within $H_{1}$ be joined to a similar point within $H_{2}$ by an arc lying within $D_{1}$. There will be a subset of this arc, an arc $R_{1} R_{2}$, such that $R_{1}$ lies on $H_{1}, R_{2}$ on $H_{2}$ and all other points of $R_{1} R_{2}$ lie without both $H_{1}$ and $H_{2}$. Then there is a simple closed curve $J_{1}$ composed of $L_{1} L_{2}+R_{1} R_{2}$ together with either arc $L_{1} R_{1}$ on $H_{1}$ and either $\operatorname{arc} L_{2} R_{2}$ on $H_{2}$. It is evident that the points of $B$ on or within $J_{1}$ that belong to $M_{1}$ can be enclosed in a finite number of circles no one of which contains or encloses a point of $L_{1} L_{2}+L_{2} R_{2}+R_{1} R_{2}+M_{2}$. And similarly those points of $B$ on or within $J_{1}$ that belong to $M_{2}$ can be enclosed in a finite number of circles no one of which contains or encloses a point of $L_{1} L_{2}+L_{1} R_{1}+R_{1} R_{2}+M_{1}$ or a point on or within a circle of the first set. Then, clearly, a point on $L_{1} L_{2}$, and therefore without $D_{1}$, could be joined to a point on $R_{1} R_{2}$, and therefore within $D_{1}$, by an arc lying within $J_{1}$ and without both these sets of circles, and therefore not containing a point of $B$. Since this leads to a contradiction we have shown that if $M$ consists of only two sets it cannot disconnect $S$. This result can evidently be extended to the case where $M$ consists of any finite number of sets.

Consider the arcs $P_{0} B_{1}$ and $P_{0} B_{2}$ above. Let $A_{2}^{\prime}$ denote the first point of $B_{2} P_{0}$ on $P_{0} B_{1}$. If $A_{2}^{\prime}$ is different from $P_{0}$ it is evident that a point on $B_{2} A_{2}^{\prime}$ very near $A_{2}^{\prime}$ can be joined to $P_{0}$ by an arc lying without $D_{1}$ and con- 
taining only $P_{0}$ in common with $P_{0} B_{1}$. From this together with $B_{2} A_{2}^{\prime}$ we obtain an arc $P_{0} B_{2}$ which lies, except for $B_{2}$, without $D_{1}$ and has only $P_{0}$ in common with $P_{0} B_{1}$. Let $M_{B_{1}}$ denote that set of $M$ to which $B_{1}$ belongs, $M_{B_{2}}$ the set to which $B_{2}$ belongs. Since $M_{B_{1}}+M_{B_{2}}$ does not disconnect $S$, $P_{0}$ can be joined to $P_{1}$ by an arc not containing any point of $M_{B_{1}}$ or $M_{B_{2}}$. Let $B_{3}$ denote the first point of $B$ on this arc in the order $P_{0} P_{1}$, and let $M_{B 3}$ denote that set of the collection $M$ to which $B_{3}$ belongs. As above, let $A_{3}^{\prime}$ denote the first point $B_{3} P_{0}$ has on the sum of the $\operatorname{arcs} P_{0} B_{1}$ and $P_{0} B_{2}$. By taking a point on $B_{3} A_{3}^{\prime}$ very near $A_{3}^{\prime}$, and drawing a suitable arc to $P_{0}$, we obtain an $\operatorname{arc} P_{0} B_{3}$ which has only $P_{0}$ in common with $P_{0} B_{1}$ or $P_{0} B_{2}$, and which lies, except for $B_{3}$, without $D_{1}$. Similarly obtain the arc $P_{0} B_{4}$ where $B_{4}$ belongs to $B$ and to $M_{B_{4}}$, such that $P_{0} B_{4}$ has only $P_{0}$ in common with $P_{0} B_{1}, P_{0} B_{2}$, or $P_{0} B_{3}$, and lies, except for $B_{4}$, without $D_{1}$. Now it is evident that the sum of two of these arcs is an arc crossing the sum of the other two. Suppose for instance that the arc $P_{0} B_{1}+P_{0} B_{3}$ crosses the arc $P_{0} B_{2}+P_{0} B_{4}$. Let $H_{3}$ be a simple closed curve (Fig. 4) enclosing $B_{1}$ but

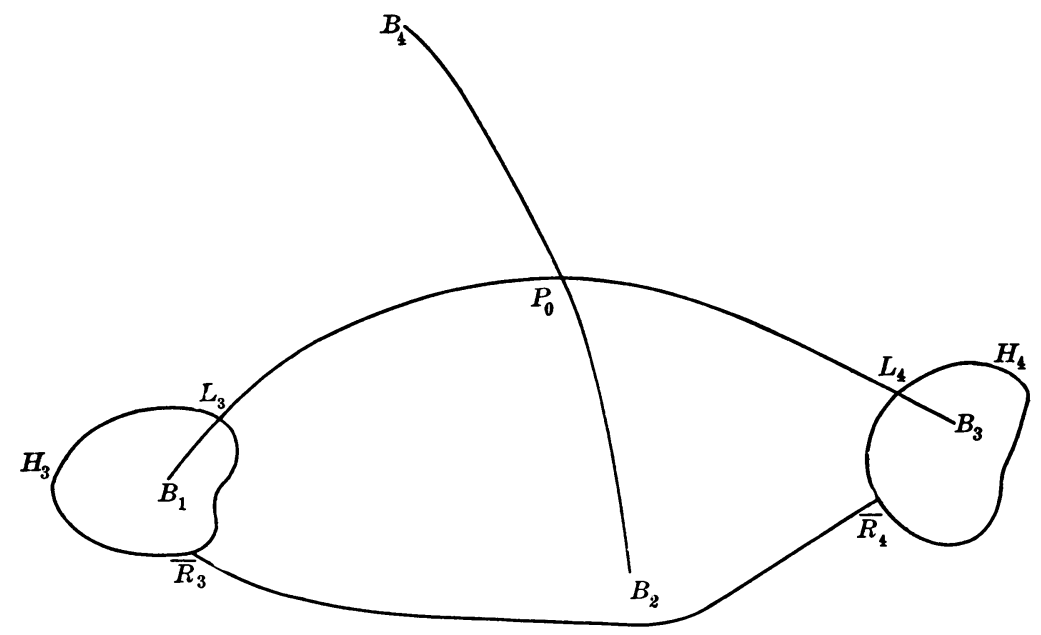

FIG. 4.

neither containing nor enclosing any point of $P_{0} B_{2}, P_{0} B_{3}, P_{0} B_{4}, M_{B_{2}}$, $M_{B 3}$, or $M_{B_{4}}$ and such that it contains only one point $L_{3}$ of the $\operatorname{arc} P_{0} B_{1}$. Let $H_{4}$ be a simple closed curve enclosing $B_{3}$, containing only one point $L_{4}$ of the $\operatorname{arc} P_{0} B_{3}$, and neither containing nor enclosing any point of $H_{3}, P_{0} B_{1}$, $P_{0} B_{2}, P_{0} B_{4}, M_{B_{1}}, M_{B_{2}}$, or $M_{B_{4}}$. Now consider the first curve of $C, C_{A}$, which has points within both $H_{3}$ and $H_{4}$. There is an arc $R_{3} R_{4}$, a subset of $C_{A}$, such that $R_{3}$ is on $H_{3}, R_{4}$ is on $H_{4}$ and all other points of $R_{3} R_{4}$ lie without both $H_{3}$ and $H_{4}$. Select one of the arcs $L_{3} R_{3}$ on $H_{3}$ and one of the arcs $L_{4} R_{4}$ on $H_{4}$ and let those selected be denoted by $L_{3} R_{3}$ and $L_{4} R_{4}$ throughout 
the discussion. Then $L_{3} L_{4}+L_{4} R_{4}+R_{3} R_{4}+L_{3} R_{3}$ is a simple closed curve $J_{2}$. Now let $\bar{R}_{3} \bar{R}_{4}$ be an arc on $C_{A}$ lying on $J_{2}$ or within $J_{2}$ except for its end points, and having $\bar{R}_{3}$ on $H_{3}$ and $\bar{R}_{4}$ on $H_{4}$ and all other points without both $H_{3}$ and $H_{4}$, such that if we consider the simple closed curve $\bar{J}_{2}$ which $\bar{R}_{3} \bar{R}_{4}$ forms with that arc of $J_{2}$ (from $\bar{R}_{3}$ to $\bar{R}_{4}$ ) that contains $L_{3} L_{4}$, there is no arc of $C_{A}$ (except $\bar{R}_{3} \bar{R}_{4}$ ) lying on $\bar{J}_{2}$ or within $\bar{J}_{2}$ except for its end points, and having one point on $H_{3}$ and one on $H_{4}$. It is possible that the $\operatorname{arc} \bar{R}_{3} \bar{R}_{4}$ will be the arc $R_{3} R_{4}$. Now it is evident that either $\underbrace{P_{0} B_{2}}_{0}{ }^{*}$ or $\underbrace{P_{0} B_{4}}_{0}$ will lie within $\bar{J}_{2}$. Suppose $P_{0} B_{2}$ does. Then $B_{2}$ will lie on or within $\overline{J_{2}}$. The point $B_{2}$ is a limit point of an infinite sequence of curves of $C$ following $C_{A}$. Let $\bar{P}_{1}, \bar{P}_{2}, \bar{P}_{3}, \ldots$ denote a sequence of points on successive curves of this sequence such that $B_{2}$ is a sequential limit point of the set $\bar{P}_{1}, \bar{P}_{2}, \bar{P}_{3}, \ldots$ If $B_{2}$ is on $\bar{J}_{2}$ these points may all coincide with $B_{2}$; if not, let them be chosen so that they lie within $\bar{J}_{2}$.

Denote by $\vec{C}_{i}$ that curve of $C$ on which $\bar{P}_{i}$ lies, and let $\bar{N}_{i}$ be the last point of $\bar{C}_{i}$ starting from $\bar{P}_{i}$ in either order around $\bar{C}_{i}$ such that $\bar{P}_{i} \bar{N}_{i}$ lies on or within $\bar{J}_{2}$. It will be seen that every $\bar{N}_{i}$ will have to be on $L_{3} \bar{R}_{3}$ or $L_{4} \bar{R}_{4}$, for if any $\bar{N}_{i}$ is on $\underbrace{\bar{R}_{3} \bar{R}_{4}}$ there must be a point $F$ of $\bar{C}_{i}$ very near $\bar{N}_{i}$, without $\bar{J}_{2}$ and therefore on the opposite side of $C_{A}$ from $P_{0} B_{2}$; for if $B_{2}$ is not on $C_{A}$ it can be joined, because of the condition put upon $\bar{R}_{3} \bar{R}_{4}$, to any point $P$ on $\bar{R}_{3} \bar{R}_{4}$ by an arc having only the point $P$ on $C_{A}$. Now since $\underbrace{P_{0}}_{0} B_{2}$ is without $C_{A}, F$ must be within $C_{A}$. This is impossible, since $F$ is on a curve following $C_{A}$ in $C$, and is therefore on or without $C_{A}$. Now the set of arcs $\bar{P}_{i} \bar{N}_{i}$ (where $\left.i=1,2,3, \cdots\right)$ determines a limit set $Y$ such that $Y$ is a closed, connected set, every point of which is on or within $\bar{J}_{2}$. Since $B_{2}$ belongs to $Y, Y$ must be a subset of $M_{B_{2}}$. But there will be a point of $Y$ on either $L_{3} \bar{R}_{3}$ or $L_{4} \bar{R}_{4}$, namely a limit point of the set of points $\bar{N}_{1}, \bar{N}_{2}, \bar{N}_{3}, \cdots$. This leads to a contradiction, for neither $H_{3}$ nor $H_{4}$ contains a point of $M_{B_{2}}$. The supposition that $M$ disconnects $S$ is therefore proved false.

Theorem 4. If $M_{1}$ and $M_{2}$ are two closed, connected, bounded point sets, neither of which disconnects a plane $S$, a necessary and sufficient condition that their sum, $M$, shall disconnect $S$ is that $\bar{M}$, the set of points common to $M_{1}$ and $M_{2}$, be not connected.

Proof. The condition is necessary. For suppose that $\bar{M}$ is connected; we can prove that $M$ does not disconnect $S$. For suppose $S-M=S_{1}+S_{2}$ where $S_{1}$ contains no limit point of $S_{2}$ nor $S_{2}$ of $S_{1}$. Let $\bar{S}$ be a square enclosing $M$. Then it is evident that either $S_{1}$ or $S_{2}$ must lie within $\bar{S}$. Suppose $S_{1}$ does. Let $P_{1}$ be a point of $S_{1}$, and consider all points which lie

* If $P_{0} B_{2}$ is an arc, $P_{0} B_{2}$ denotes the point set $P_{0} B_{2}-P_{0}-B_{2}$. 
with $P_{1}$ in a connected subset of $S_{1}$. It will be seen that since $M$ is closed, these points form a domain $D_{s}$, a subset of $S_{1}$, and of the interior of $\bar{S}$, such that the boundary of $D_{s}$ is a subset of $M$. Let $M^{\prime}$ denote the point set $M_{1}-\bar{M}_{2}$ and $M^{\prime \prime}$ the point set $M_{2}-\bar{M}$. Let $P_{2}$ denote a point of $S_{2}$ that is without $\bar{S}$; by the lemma, $P_{2}$ can be joined to $P_{1}$ by an arc not containing any point of $M_{2}$. Let $P^{\prime}$ denote the first point the arc $P_{2} P_{1}$ has on

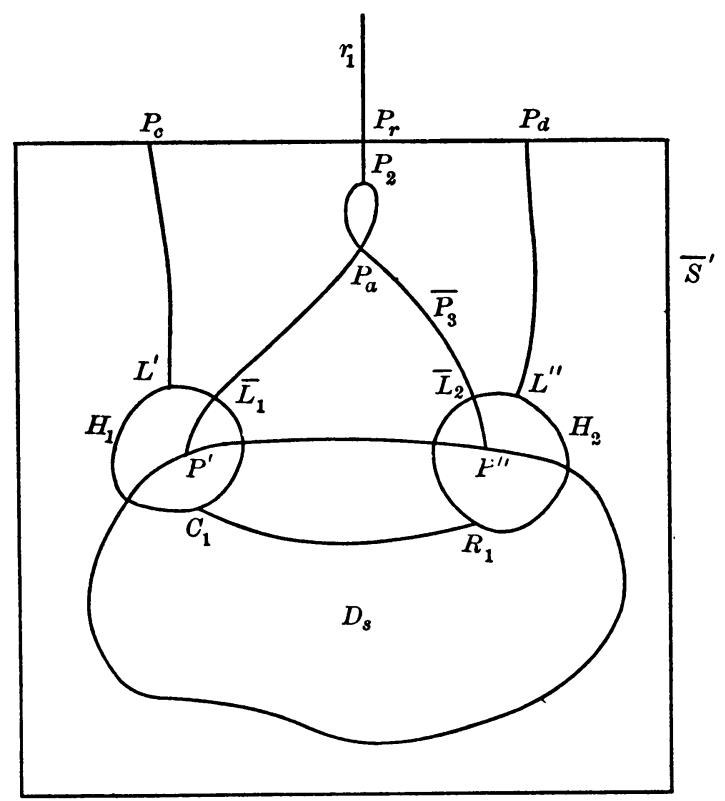

Fig. 5.

the boundary of $D_{s}$ (Fig. 5), let $P_{2}$ be joined to $P_{1}$ by an arc not containing any point of $M_{1}$, and let $P^{\prime \prime}$ denote the first point of this arc $P_{2} P_{1}$ on the boundary of $D_{s}$. Then $P^{\prime}$ belongs to $M^{\prime}$ and $P^{\prime \prime}$ to $M^{\prime \prime}$. Then there exists an $\operatorname{arc} P^{\prime} \bar{P}_{3} P^{\prime \prime}$, a subset of $P_{2} P^{\prime}+P_{2} P^{\prime \prime}$, which lies, except for its end points, without $D_{s}$. As in Theorem 3 , there exists a simple closed curve $H_{1}$ enclosing $P^{\prime}$, containing only one point $\bar{L}_{1}$ of the $\operatorname{arc} P^{\prime} \bar{P}_{3} P^{\prime \prime}$, and neither containing nor enclosing a point of $M_{2}$, and there exists a corresponding curve $H_{2}$ enclosing $P^{\prime \prime}$, containing only one point $\bar{L}_{2}$ of the arc $P^{\prime} \bar{P}_{3} P^{\prime \prime}$ and neither containing nor enclosing a point of $M_{1}$ or $H_{1}$, and there exists an $\operatorname{arc} C_{1} R_{1}$ in $D_{s}$ having only $C_{1}$ on $H_{1}$ and $R_{1}$ on $H_{2}$. Then there is a simple closed curve $\bar{J}_{1}$, composed of $\bar{L}_{1} \bar{P}_{3} \bar{L}_{2}+C_{1} R_{1}$ together with the arcs $\bar{L}_{1} C_{1}$ on $H_{1}$ and $\bar{L}_{2} R_{1}$ on $H_{2}$ so chosen that $\bar{J}_{1}$ encloses $P^{\prime}$ and $P^{\prime \prime}$.

We can show as in Theorem 3 that if those points of the boundary of $D_{s}$ that this curve contains or encloses belong to $M^{\prime}+M^{\prime \prime}$ then two points, one without $D_{s}$ and the other within $D_{s}$, can be joined by an arc lying within 
$\bar{J}_{1}$ and containing no point of the boundary of $D_{s}$. Since this is impossible $\bar{J}_{1}$ must enclost some point of $\bar{M}$, and since $\bar{M}$ is connected and since no point of $\bar{M}$ is on $\bar{J}_{1}, \bar{M}$ must lie wholly within $\bar{J}_{1}$.

A ray of a straight line can be drawn from $P_{2}$, lying wholly without $\bar{S}$, and there exists a ray $r_{1}$, a subset of the first ray together with one of the arcs $P_{2} P_{1}$, such that $r_{1}$ lies without $\bar{J}_{1}$ except for its end point $P_{a}$ on $P^{\prime} \bar{P}_{3} P^{\prime \prime}$. Let $\bar{S}^{\prime}$ denote a square enclosing $\bar{S}$ and both arcs $P_{1} P_{2}$ and containing only one point $P_{r}$ of $r_{1}$. If $L^{\prime}$ is a point on $H_{1}$ and $\bar{J}_{1}$ sufficiently near $\bar{L}_{1}$ there exists an $\operatorname{arc} P_{c} L^{\prime}$ such that $P_{c}$ is a point on $\overline{S^{\prime}}$ and such that $P_{c} L^{\prime}$ lies without $D_{s}, P_{c} L^{\prime}-L^{\prime}$ lies without $\bar{J}_{1}$ and $P_{c} L^{\prime}-P_{c}$ lies within $\bar{S}^{\prime}$. Similarly a point $L^{\prime \prime}$ of $H_{2}$ and $\bar{J}_{1}$ can be joined by an arc to the point $P_{D}$ of $\bar{S}^{\prime}$, and in such a way that the $\operatorname{arc} P_{D} L^{\prime \prime}$ contains no point of the $\operatorname{arc} P_{c} L^{\prime}$. Let $L^{\prime} L^{\prime \prime}$ denote the arc composed of $P_{c} L^{\prime}+P_{D} L^{\prime \prime}$ together with that arc of $\overline{S^{\prime}}$, from $P_{c}$ to $P_{D}$, which does not contain $P_{r}$. The arc $L^{\prime} L^{\prime \prime}$ together with the $\operatorname{arc} L^{\prime} C_{1} R_{1} L^{\prime \prime}$ of $\bar{J}_{1}$ makes a simple closed curve $\bar{J}_{2}$ whose interior has no point in common with that of $\bar{J}_{1}$. Since $\bar{J}_{2}$ encloses points of $D_{s}$ we can show as above that it must contain all of $\bar{M}$. Since this is obviously impossible, the supposition that $\bar{M}$ was connected is proved to be false.

The condition is sufficient. For suppsoe $\bar{M}$ is not connected. Then it is the sum of two closed, mutually exclusive point sets, $\bar{M}_{1}$ and $\bar{M}_{2}$. We can show that in this case $S-M=S_{1}+S_{2}$ where $S_{1}$ and $S_{2}$ are mutually exclusive and neither contains a limit point of the other.

The point set $\bar{M}_{1}$ contains some point $\bar{P}_{1}$ which is a limit point of $M^{\prime \prime}$ since $M_{2}$ is connected and $\bar{M}_{1}$ and $\bar{M}_{2}$ are closed point sets. Let $J_{p_{1}}$ denote a circle enclosing $\bar{P}_{1}$ but neither containing nor enclosing any point of $\bar{M}_{2}$. This circle contains a point $P_{1}^{*}$ of $M_{2}-\bar{M}_{1}-\bar{M}_{2}$, and there is a ray of an open curve from $P_{1}^{*}$ not containing any point of $M_{1}$, since $M_{1}$ is closed and bounded and does not disconnect $S$. And a subset of this ray is a ray $r_{2}$, having an end point on $J_{p_{1}}$ and lying, except for this end point, without $J_{p_{1}}$. Now, by the Heine-Borel theorem, $\bar{M}_{2}$ can be enclosed in a finite set of circles no one of which contains or encloses any point of $\bar{M}_{1}$ or $J_{p_{1}}$ or $r_{2}$. Consider any one of these circles together with all of the set that are connected with it. By Theorem 42 of The foundations $†$ there exists a simple closed curve $J_{c_{2}}$, a subset of these circles, whose interior contains all of their interiors. It will not contain any point of $\bar{M}$, but will enclose some points of $\bar{M}$; let $M_{2}^{*}$ denote the set of these points. The curve $J_{p_{1}}$ with $r_{2}$ will be without $J_{c_{2}}$. Now, as above, there exists a ray $r_{3}$ which has its end point on $J_{c_{3}}$, lies, except for this point, without $J_{c_{2}}$ and contains no point of $M_{1}$. Then all points of $\bar{M}$ that are without $J_{c_{2}}$ can be enclosed in a finite set of circles no one of which contains or encloses any point of $J_{c_{2}}$ or $r_{3}$. If these circles do not form a connected

† Cf. R. L. Moore, loc. cit., p. 156. 
point set they can be joined by a finite number of arcs not containing any point of $J_{c_{2}}+r_{3}$. For $J_{c_{2}}$ with its interior does not disconnect $S$, and $r_{3}$ does not disconnect $S$ and they have only one point in common; therefore, by the first part of this theorem, $J_{c_{2}}$ with its interior and $r_{3}$ does not disconnect $S$. These arcs can be covered by a finite set of circles not containing any point of $J_{c_{2}}$ or $r_{3}$, and hence from all these circles a simple closed curve $J_{c_{1}}$ can be obtained which has the following properties: it is wholly without $J_{c_{2}}$ and does not enclose it; it encloses all points $M_{1}^{*}$, of $\bar{M}$, that $J_{c_{2}}$ does not enclose; therefore it contains no point of $\bar{M}$.

The points of $M_{2}$ that are on $J_{c_{1}}$ or $J_{c_{2}}$ or without both $J_{c_{1}}$ and $J_{c_{2}}$ form a closed set, a subset of $M^{\prime \prime}$. This set can be covered by a finite set of circles, $T$, such that no circle of $T$ contains or encloses any point of $M_{1}$. Now there is a ray of an open curve, not containing any point of $M_{1}$, from some point within each of these circles. Let $\left[r_{t}\right]$ denote the sum of such rays. And let $M_{1}$ be covered by a finite set of circles no one of which contains or encloses any point of the circles $T$, or of the point set $\left[r_{t}\right]+r_{2}+r_{3}$. Since $M_{1}$ is connected this set of circles will be connected, and, as above, there exists a simple closed curve $J_{1}$ which is a subset of them and encloses all of their interiors and therefore all of $M_{1}$. The curve $J_{1}$ cannot wholly enclose either $J_{c_{1}}$ or $J_{c_{2}}$ since it contains no point of $r_{2}$ or $r_{3}$.

Let $S^{*}$ be a square enclosing $J_{c_{1}}, J_{c_{2}}, J_{1}$ and $M_{2}$. It is evident that there is an arc $P_{3} P_{4}$ composed of a finite number of straight line intervals lying within $S^{*}$, except for the points $P_{3}$ and $P_{4}$ which are on $S^{*}$, and which separates the interior of $S^{*}$ into two parts such that $J_{c_{1}}$ lies wholly within one part and $J_{c_{2}}$ within the other. Let $A$ be a point of $\bar{M}$ within $J_{c_{1}}$ and $B$ a point of $\bar{M}$ within $J_{c_{2}}$. By Theorem 1 of this paper there is a connected subset of $M_{2}$ lying within $J_{1}$ such that $A$ and some point $A^{\prime}$ of $J_{1}$ are limit points of it. Moreover this set will lie within $J_{c_{1}}$ since it is connected, $A$ lies within $J_{c_{1}}$ and $J_{1}$ encloses no point of $M_{2}$ that is on $J_{c_{1}}$. Similarly there exists a connected subset of $M_{2}$ lying within $J_{1}$ and $J_{c_{2}}$ such that $B$ and some point $B^{\prime}$ on $J_{1}$ are limit points of it.

Now there is a subset, $C D$, of $P_{3} P_{4}$ which satisfies the following conditions: it is an arc lying except for $C$ and $D$ within $J_{1} ; C$ and $D$ are on $J_{1}$ and separate $A^{\prime}$ and $B^{\prime}$ on $J_{1}$. For consider all arcs of $P_{3} P_{4}$ which lie except for their end points within $J_{1}$ and have their end points on $J_{1}$. There are a finite number of these, since $J_{1}$ is composed of a finite number of arcs of circles and $P_{3} P_{4}$ of a finite number of straight line intervals. Let $L_{1}, L_{2}, L_{3}, \cdots$, denote the set of all such arcs and let $L$ denote the point set $L_{1}+L_{2}+L_{3} \cdots$. Suppose that no $L_{j}$ separates $A^{\prime}$ and $B^{\prime}$ on $J_{1}$. Denote by $a^{\prime}$ and $a^{\prime \prime}$ the two $\operatorname{arcs} A^{\prime} B^{\prime}$ on $J_{1}$. If any arc $L_{i}$ of $L$ has an end point on $a^{\prime}$ for instance let $X_{1}$ denote the first such end point on $a^{\prime}$ in the order $A^{\prime} B^{\prime}$ and $X_{2}$ the other 
end point of this arc. Then consider the simple closed curve $X_{1} A^{\prime}+a^{\prime \prime}$ $+B^{\prime} X_{2}+X_{2} X_{1}$. It will be seen that $A^{\prime}$ and $B^{\prime}$ can be joined by an arc lying on or within this curve which contains no point of $L_{i}$. By a finite number of repetitions of this process it is evident that we can obtain a simple closed curve $C_{x}$, a subset of $J_{1}$ plus its interior, containing $A^{\prime}$ and $B^{\prime}$ but enclosing no point of $L . \quad A^{\prime}$ and $B^{\prime}$ could then be joined by an arc lying on or within $C_{x}$ and containing no point of $L$ and hence no point of $P_{3} P_{4}$. Since this is impossible there must exist an arc $C D$, having the properties stated above.

Since $C D$, being a subset of $P_{3} P_{4}$, lies without both $J_{c_{1}}$ and $J_{c_{2}}$ and since it lies within $J_{1}$, it contains no point of $M_{2}$. Therefore of the two regions into which it divides the interior of $J_{1},{ }^{*}$ one must contain $A$, the other $B$. Let $R$ denote the interior of $J_{1}$. Let $F_{1} F_{2} F_{3}$ be an arc as indicated in Fig. 6

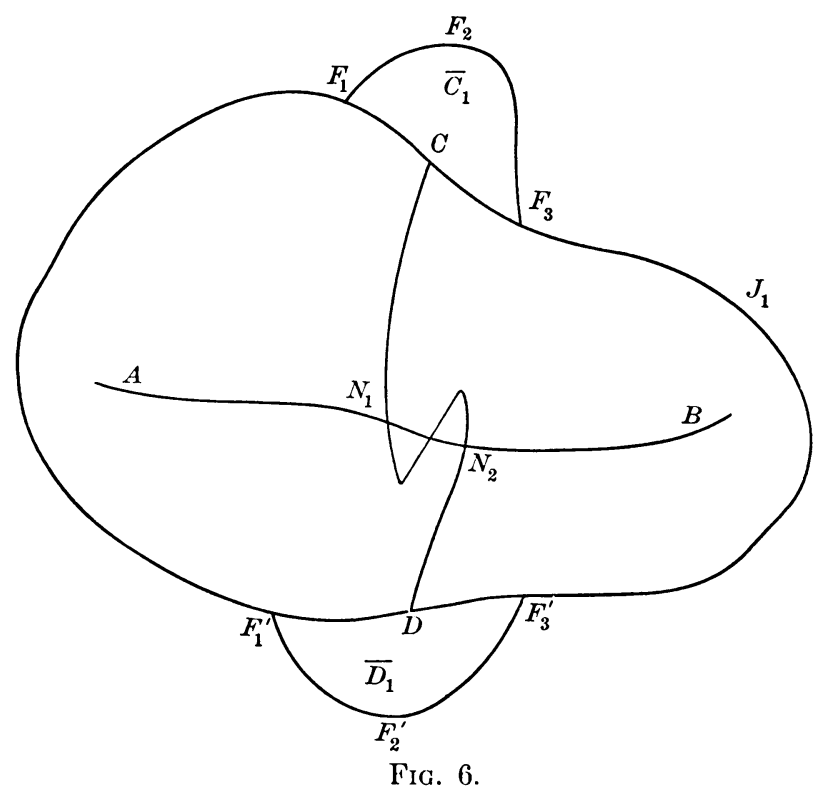

with its end points on $J_{1}$ and lying, except for its end points, without $J_{1}$ and wholly without $J_{c_{1}}$ and $J_{c_{2}}$ and such that the simple closed curve $F_{1} C F_{3} F_{2} F_{1}$ neither contains nor encloses a point of $M_{1}$ or $M_{2}$. Let $\bar{C}_{1}$ be a point within this curve. Let $F_{1}^{\prime} F_{2}^{\prime} F_{3}^{\prime}$ be a similar arc forming part of a simple closed curve $F_{1}^{\prime} D F_{3}^{\prime} F_{2}^{\prime} F_{1}^{\prime}$ and let $\bar{D}_{1}$ be a point within it. Now if we suppose that $M$ does not disconnect $S$, let $N_{1}$ be the first point of the $\operatorname{arc} C D$ on $M_{1}$. Then by the first part of this theorem, $C N_{1}+M$ does not disconnect $S$. Let $N_{2}$ be the first point the arc $D C$ has on $M_{1}\left(N_{1}\right.$ and $N_{2}$ must exist since $M_{1}$ is con-

* Cf. R. L. Moore, loc. cit., p. 141. 
nected and $C D$ divides $R$ into two parts, of which one contains $A$, and the other contains $B$ ). By several applications of the first part of this theorem, we can show that $M+C N_{1}+D N_{2}+F_{1} C F_{3}+F_{1}^{\prime} D F_{3}^{\prime}$ does not disconnect $S$. Let $H$ denote this point set. Then $\bar{C}_{1}$ and $\bar{D}_{1}$ can be joined by a broken line containing no point of $H$. By extending this broken line we get a broken line $C E D$ which has only $C$ and $D$ on $H$. By Theorem 43 of The founddtions $\dagger$ there is a simple closed curve $\bar{C}$, a subset of $J_{1}$ together with the broken line $C E D$ whose interior is a subset of $R$, which encloses $N_{1}$ but no point of the arc $C E D$. Then $\bar{C}$ will enclose all of $M_{1}+\left(C N_{1}-C\right)+\left(D N_{2}-D\right)$, since this is a connected point set and has no point on $\bar{C}$. The curve $\bar{C}$ will contain $F_{1} C F_{3}, F_{1}^{\prime} D F_{3}^{\prime}, A^{\prime}$ and $B^{\prime}$ since it encloses $A$ and $B$ and contains no point of the previously described connected sets of $M_{2}$ between $A$ and $A^{\prime}$, and $B$ and $B^{\prime}$. Then it is evident that there is a curve $\bar{C}^{\prime}$, which has all the above mentioned properties of $\bar{C}$ except that it contains only the points $C$ and $D$ of the broken line $C E D$ and which is such that (1) it is a subset of $\bar{C}$ plus its interior, (2) those points of $\bar{C}^{\prime}$ that are not on $\bar{C}$ do not belong to $M$.

It is possible that the broken line segment $C D-C-D$ (a subset of $\left.P_{3} P_{4}\right)$ will not lie within $\bar{C}^{\prime}$. Consider the arcs of the broken line $C D$ which lie except for their end points without $\bar{C}^{\prime}$ and have their end points on $\bar{C}^{\prime}$. Let $C^{\prime} D^{\prime}$ denote such an arc. $C^{\prime}$ and $D^{\prime}$ are points of $R$. Let $E^{\prime}$ denote a point of $C^{\prime} D^{\prime}$ without $\bar{C}^{\prime}$. The points $C^{\prime}$ and $D^{\prime}$ can be joined by an arc $C^{\prime} F^{\prime} D^{\prime}$ such that $C^{\prime} F^{\prime} D^{\prime}$ lies within $\bar{C}^{\prime}$ and therefore within $J_{1}$. Then the simple closed curve $C^{\prime} E^{\prime} D^{\prime} F^{\prime} C^{\prime}$ is a subset of $R$, and therefore the $\operatorname{arc} C^{\prime} G D^{\prime}$ of $\bar{C}^{\prime}$ that it encloses must be a subset of $R$, and therefore it must belong to that part of $\bar{C}^{\prime}$ which does not belong to $\bar{C}$ and which therefore contains no points of $M$. Now there are only a finite number of arcs such as $C^{\prime} E^{\prime} D^{\prime}$, that are parts of the broken line $C D$, which lie except for their end points without $\bar{C}^{\prime}$ and have their end points on $\bar{C}^{\prime}$, and we see that each one can be replaced by an arc lying on $\bar{C}^{\prime}$ and containing no point of $M_{2}$. This gives us a continuous curve from $C$ to $D$ lying on or within $\bar{C}^{\prime}$ and containing no point of $M_{2}$, and there is an arc, a subset of this curve, having the same properties. It is evident that this arc can be replaced by an arc $C E^{*} D$ lying within $\bar{C}^{\prime}$ except for $C$ and $D$ and containing no point of $M_{2}$. Now $C$ and $D$ separate $A^{\prime}$ and $B^{\prime}$ on $\bar{C}^{\prime}$. For if they did not, $A^{\prime}$ and $B^{\prime}$ could be joined by an arc $a_{1}$ lying, except for $A^{\prime}$ and $B^{\prime}$, within $\bar{C}^{\prime}$, and $C$ and $D$ could be joined by a similar arc $a_{2}$ such that $a_{2}$ had no point in common with $a_{1}$. But both these arcs would lie except for their end points within $\bar{C}$ and $J_{1}$ and have their end points on $\bar{C}$ and $J_{1}$. This leads to a contradiction, since $C$ and $D$ separate $A^{\prime}$ and $B^{\prime}$ on $J_{1}$.

Now the arc $C E D$, together with the arc $C E^{*} D$, forms a simple closed † See R. L. Moore, loc. cit., p. 157. 
curve $J_{3}$ containing no point of $M_{2}$, but such that one of the points $A$ and $B$ is within it, and the other without it. This is impossible since $M_{2}$ is a connected point set. Therefore the supposition that $\bar{C}_{1}$ and $\bar{D}_{1}$ can be joined by an arc containing no point of $H$ is false and since $H$ disconnects $S, M$ must disconnect $S$. Evidently one of the two sets into which $M$ separates $S$ is the point set, $S_{1}$, composed of $\bar{C}_{1}$ together with all points that can be joined to it by arcs containing no point of $M$. The set $S_{2}$ will then be the point set $S-M-S_{1}$.

Theorem 5.* If $M_{1}$ and $M_{2}$ are two closed, bounded, connected point sets in a plane $S$, such that neither $M_{1}$ nor $M_{2}$ disconnects $S$ and such that $M_{1}$ and $M_{2}$ have in common only $K_{1}$ and $K_{2}$, where $K_{1}$ and $K_{2}$ are mutually exclusive connected sets, then $S-M_{1}-M_{2}$ is the sum of just two mutually exclusive, connected domains.

Proof. We have shown above that under the conditions of this theorem $S-M_{1}-M_{2}$ is not connected. Suppose then that it is the sum of more than two mutually exclusive connected domains. There will exist three points, $P_{1}, P_{2}$ and $P_{3}$, no two of which can be joined by an arc containing no point of $M_{1}+M_{2}$. It is evident that in the preceding theorem the curve $J_{1}$ could have been constructed in such a way that $P_{1}, P_{2}$ and $P_{3}$ were without it; for there exist three open curves, containing $P_{1}, P_{2}$ and $P_{3}$ but containing no point of $M_{1}$, and $J_{1}$ could have been drawn so as not to contain any point of these open curves. We shall suppose that $J_{1}$ has been so drawn. We can furthermore suppose that $J_{1}$ is replaced by a polygon $W$, satisfying the conditions which $J_{1}$ satisfies.

Now $P_{1}$ and $P_{2}$ can be joined by an arc made up of a finite number of straight line intervals containing no point of $M_{2}$, since $M_{2}$ does not disconnect $S$; and since $M_{2}+P_{1} P_{2}$ does not disconnect $S$, it is obvious that $P_{2}$ and $P_{3}$ can be joined by a similar arc which contains no point of $M_{2}$ and has only $P_{2}$ in common with $P_{1} P_{2}$. Similarly there is a broken line, an $\operatorname{arc} P_{3} P_{1}$, containing no point of $M_{2}$, and having only $P_{3}$ and $P_{1}$ in common with $P_{1} P_{2}+P_{2} P_{3}$. Now any one of these three arcs will contain only a finite number of intervals lying except for their end points in $W$ and having their end points on $W$. These intervals will be of two kinds, those whose end points separate $A^{\prime}$ and $B^{\prime}$ on $W$, and those whose end points do not separate $A^{\prime}$ and $B^{\prime}$ on $W$. Consider an interval $\bar{X}_{1} \bar{X}_{2}$ of the second sort. Let $\bar{X}_{1} X^{\prime} \bar{X}_{2}$ denote that arc on $W$ which does not contain $A^{\prime}$ or $B^{\prime}$. Then obviously $\bar{X}_{1} \bar{X}_{2}+\bar{X}_{1} X^{\prime} \bar{X}_{2}$ is a simple closed curve enclosing no point of $K_{1}$ or $K_{2}$, and by the method employed in Theorem 3, we can draw an arc $\bar{X}_{1} \bar{X}_{2}$ such that $\bar{X}_{1} \bar{X}_{2}$ is within this curve and contains no point of either

$\therefore$ * Rosenthal gives a proof for the case in which each of the sets $K_{1}$ and $K_{2}$ reduces to a single point. See A. Rosenthal, loc. cit., p. 102, Theorem 6 . 
$M_{1}$ or $M_{2}$. Let the original interval $\bar{X}_{1} \bar{X}_{2}$ be replaced by this arc $\bar{X}_{1} \bar{X}_{2}$ and let this process be carried out for every such interval, of the second kind, on each of the three arcs. Now each one of the three must have at least one interval of the first kind; for since no one of these arcs has any point of $M_{2}$ on it and all of $M_{1}$ lies within $W$ and every interval of the second sort of the arcs lying within $W$ has been replaced by an arc containing no point of either $M_{1}$ or $M_{2}$, if one of these arcs, $P_{1} P_{2}$, for instance, had no interval of the first sort on it, it would be replaced by an arc $P_{1} P_{2}$ which had no point of $M_{1}$ or $M_{2}$ on it, which is contrary to our supposition. Let $Y_{1} Y_{2}$ be the first interval on $P_{1} P_{2}$ in the order $P_{1} P_{2}$ which separates $A^{\prime}$ and $B^{\prime}$ on $W$, let $Y_{3} Y_{4}$ be a similar arc on $P_{2} P_{3}$, and let $Y_{5} Y_{6}$ be a similar arc on $P_{3} P_{1}$. Some two of the points $Y_{1}, Y_{3}, Y_{5}$ must lie on the same arc $A^{\prime} B^{\prime}$ of $W$; suppose $Y_{1}$ and $Y_{3}$ do (Fig. 7). Now consider the simple closed curve

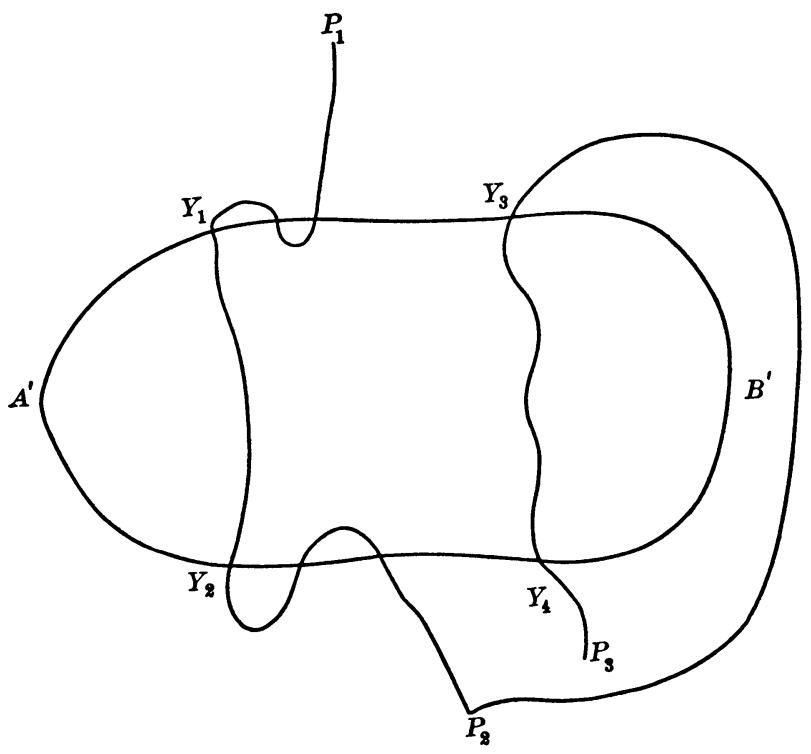

FIg. 7.

$\bar{Y}=Y_{1} Y_{3} Y_{4} Y_{2} Y_{1}$. Since it contains neither $A^{\prime}$ nor $B^{\prime}$ it encloses no point of either $K_{1}$ or $K_{2}$. Now there is no connected subset of $M_{2}$ lying on or within $\bar{Y}$ and having a point $\bar{X}_{3}$ on $Y_{1} Y_{3}$ and a point $\bar{X}_{4}$ on $Y_{2} Y_{4}$. For such a set would separate $A^{\prime}$ and $B^{\prime}$ on $W$ and would divide the interior of $W$ into two sets of which one contains $K_{1}$ and the other contains $K_{2}$. But $K_{1}$ and $K_{2}$ are connected within $W$ by a set of points belonging to $M_{1}$. This set would have to cross the subset of $M_{2}$ lying within $Y$. But this is obviously impossible. Then by Theorem 2 there is a division of the points of $M_{2}$ that lie on or within $\bar{Y}$ into two mutually exclusive closed sets, $Z_{1}$ and $Z_{2}$, such that $Z_{1}$ 
contains all the points of $M_{2}$ that lie on $Y_{1} Y_{3}$. Now all points of $Z_{1}$ can be enclosed in a finite number of circles not containing any point of $Y_{3} Y_{4}+Y_{4} Y_{2}$ $+Y_{2} Y_{1}$ or any point of $Z_{2}$ or $M_{1}$, and evidently there is an arc lying on or within $\bar{Y}$, a subset of these circles together with intervals of $Y_{1} Y_{3}$, which contains no point of either $M_{1}$ or $M_{2}$. Then $P_{1}$ and $P_{2}$ are joined by an arc not containing any point of either $M_{1}$ or $M_{2}$.- Since this is contrary to our supposition the theorem is proved.

UNIVERSITY OF Texas, Austin, Tex. 